\title{
Analysis of plasma miR-208a and miR-370 expression levels for early diagnosis of coronary artery disease
}

\author{
HONGSHENG LIU ${ }^{1}$, NING YANG ${ }^{2}$, ZHONGHUA FEI $^{1}$, JIE QIU $^{1}$, DONGWEN MA ${ }^{1}$, \\ XINMEI LIU ${ }^{1}$, GUOQIANG CAI ${ }^{1}$ and SHENG $\mathrm{LI}^{3}$ \\ ${ }^{1}$ Cardiac Intensive Care Unit and ${ }^{2}$ Center of Health Management and Community Service, \\ The Affiliated Hospital of Jining Medical University, Jining, Shandong 272029; \\ ${ }^{3}$ Department of Biochemistry, Dalian Medical University, Dalian, Liaoning 116044, P.R. China
}

Received April 1, 2016; Accepted July 7, 2016

DOI: $10.3892 /$ br.2016.726

\begin{abstract}
Coronary artery disease (CAD) requires more accurate diagnostic methods, for which circulating microRNAs (miRNAs) are promising non-invasive biomarkers. miR-208a and miR-370 are two key molecules in cardiac hemostasis and lipid metabolism, respectively. The aim of the present study was to evaluate their potency as diagnostic biomarkers for CAD. Plasma miR-208a and miR-370 were quantitated by reverse transcription-quantitative polymerase chain reaction (RT-qPCR) using a TaqMan ${ }^{\circledR}$ MicroRNA Reverse Transcription and PCR kit in 95 CAD patients and 50 non-CAD control subjects. The association between the miRNA levels and CAD was analyzed statistically. The plasma levels of miR-208a $(\mathrm{P}=0.006)$ and miR-370 $(\mathrm{P}=0.003)$ were significantly higher in the CAD group than in the control group. Using receiver operating characteristic analysis it was shown that the area under the curve (AUC) of miR-208a and miR-370 was 0.819 and 0.745 , respectively. The combination of miR-208a and miR-370 exhibited the largest AUC of 0.856 . Thus, miR-208a and miR-370 are promising diagnostic biomarkers for discriminating CAD and may facilitate the management of patient care. The combination of the two miRNAs may be more efficacious than either miRNA alone for the diagnosis of CAD.
\end{abstract}

\section{Introduction}

Coronary artery disease (CAD), traditionally regarded as a disease of Western society, is currently the leading cause of morbidity and mortality in China (1). A marked increase in the mortality rate of coronary heart disease is the major reason for the long-term rise of cardiovascular disease (CVD)-associated

Correspondence to: Dr Hongsheng Liu, Cardiac Intensive Care Unit, The Affiliated Hospital of Jining Medical University, 79 Guhuai Road, Jining, Shandong 272029, P.R. China

E-mail: 18678766889@163.com

Key words: miR-208a, miR-370, coronary heart disease, early diagnosis fatalities in the Chinese population (1). CAD is a slowly progressive disease that exhibits few symptoms at its early stage when prevention is possible, thus, non-invasive approaches to establish the severity of CAD is of great importance to patient care.

MicroRNA (miRNA) is a class of small (19-25 nt) single-stranded non-coding RNAs that post-transcriptionally regulate gene expression and are crucial in a wide range of biological processes, such as metabolism, differentiation, proliferation and apoptosis (2-4). It has been found that miRNA levels are tissue-specific and closely associated with physiological/pathological changes in the cardiovascular system (5). In addition, due to their small size, miRNAs detected in body fluids are stable and resistant to digestion of endogenous RNases (6). These characteristics render miRNAs as ideal biomarkers for CVD.

In recent years there have been numerous studies on the potential role of miRNAs as diagnostic biomarkers in the field of CVD. miR-499 and miR-208a/b belong to the miR-208 family, which is almost specifically expressed in the heart. The expression levels of these three miRNAs have been found to be promptly increased to a significant extent in the circulation following acute myocardial infarction (MI) and successfully discriminate MI, more effectively than the traditional biomarker, cardiac troponin (7-9). In the early stages of CAD, although various miRNAs (for example, miR-21, miR-145, miR-199a and miR-340) were found to be deregulated (10-14), the findings were not conclusive. The performance of certain miRNAs was inconsistent between different studies. Thus, more cohort studies are required for further validation.

In the present study, two plasma miRNAs (miR-208a and miR-370) that are vital in the progression and development of CAD were evaluated by reverse transcription-quantitative polymerase chain reaction (RT-qPCR) in a Chinese cohort of 95 CAD patients and 50 non-CAD control subjects. Their association with CAD was investigated, and their ability to discriminated CAD was assessed using receiver operating characteristic (ROC) analysis.

\section{Materials and methods}

Subjects. The ethics committee of the Affiliated Hospital of Jining Medical University (Jining, China) approved the present 
study, and written consent was obtained from all patients to provide information and samples for research purposes.

Between July 21, 2005 and March 7, 2015, 95 consecutive patients (65 males and 30 females; age range, 44-78 years) who underwent diagnostic coronary angiography for chest pain evaluation were recruited from inpatients admitted to the Affiliated Hospital of Jining Medical University. Diagnosis of CAD was confirmed by quantitative coronary angiography and was defined as angiographic evidence of $>50 \%$ luminal narrowing in at least one segment of a main epicardial coronary artery. Two cardiologists, blinded to which patients were in the study, assessed the angiogram. Fifty individuals with neither detectable coronary stenosis nor atherosclerotic vascular disease were considered as non-CAD patients, who were matched for age, gender, smoking habits, hypertension and diabetes. Patients with histories of significant concomitant diseases, including cardiomyopathy, congenital heart disease, hepatic failure, renal failure, hepatitis, bleeding disorders, and malignant diseases were excluded from the study.

Sample collection and plasma isolation. A sample of fasting blood ( $\sim 5 \mathrm{ml})$ was withdrawn in ethylenediaminetetraacetic acid-containing tubes and immediately centrifuged at $4^{\circ} \mathrm{C}$ for $10 \mathrm{~min}$ at $1,000 \mathrm{x}$ g. Plasma was collected and stored at $-80^{\circ} \mathrm{C}$ in a freezer until RNA purification.

RNA purification and miRNA quantification. RNA was extracted from $800 \mu \mathrm{l}$ plasma using an miRNeasy serum/plasma kit (Qiagen China, Co., Ltd., Shanghai, China) according to the manufacturer's instructions. The RNA was eluted in $50 \mu \mathrm{l}$ nuclease free water and $40 \mathrm{ng}$ RNA (in $5 \mu \mathrm{l}$ ) was reverse transcribed with TaqMan ${ }^{\circledR}$ MicroRNA Reverse Transcription kit (Thermo Fisher Scientific, Inc., Waltham, MA, USA) according to the manufacturer's instructions. cDNA was quantitated using a TaqMan-based RT-qPCR assay specific for miR-208a and miR-370 according to the manufacturer's recommended protocol on an Applied Biosystems 7500 Sequence Detection System (the miR-208a and miR-370 specific primers for RT and PCR were enclosed in the kits). The cycling conditions were $95^{\circ} \mathrm{C}$ for $10 \mathrm{~min}$ followed by 40 cycles at $95^{\circ} \mathrm{C}$ for $15 \mathrm{sec}$ and $60^{\circ} \mathrm{C}$ for $1 \mathrm{~min}$. All reactions were performed in triplicate. The expression levels of circulating miRNAs are presented as the quantitation cycle $(\mathrm{Cq})$ values. Exogenously added cel-miR-39 served as a control for normalizing the data. The comparative $\mathrm{Cq}(\Delta \mathrm{Cq})$ method was used to analyze the miRNA expression levels (15).

Statistical analysis. Statistical analysis between numerical variables of the two groups was performed using unpaired Student's t-test for parametric data or Mann-Whitney rank sum test for nonparametric data. $\mathrm{P}<0.05$ was considered to indicate a statistically significant difference. Logistic regression was conducted to construct ROC curves using the expression level of miRNA adjusted for the matching factors. The area under the curve (AUC) was calculated with a $95 \%$ confidence interval (CI). The specificity and sensitivity were identified by numerical integration of each ROC curve. A prediction model was constructed by fitting miRNAs into a logistic regression model, and the stepwise backward model was used to determine miRNA combinations in discriminating CAD
Table I. Clinical characteristics of study subjects.

\begin{tabular}{lccc}
\hline & $\begin{array}{c}\text { CAD } \\
\text { patients, } \\
\mathrm{n}=95\end{array}$ & $\begin{array}{c}\text { Non-CAD } \\
\text { subjects, } \\
\mathrm{n}=50\end{array}$ & P-value \\
\hline Aariables & $65(44-78)$ & $65(46-75)$ & 0.953 \\
Male/female & $65 / 30$ & $34 / 16$ & 0.613 \\
BMI (kg/m²) & $23(20-26)$ & $22(20-24)$ & 0.726 \\
Active smoker $(\%)$ & 63.6 & 61.0 & 0.824 \\
Diabetes mellitus $(\%)$ & 9.6 & 9.1 & 0.823 \\
Hypertension $(\%)$ & 52.3 & 50.8 & 0.208 \\
Systolic BP (mmHg) & $131(98-185)$ & $125(112-145)$ & 0.349 \\
Diastolic BP (mmHg) & $82(56-97)$ & $81(62-87)$ & 0.561 \\
Left ventricular & $58(38-68)$ & $61(50-65)$ & 0.145 \\
ejection fraction $(\%)$ & & & \\
\hline
\end{tabular}

Data are presented as the median with the range in parentheses. $\mathrm{CAD}$, coronary artery disease; $\mathrm{BP}$, blood pressure.

patients with hypercholesterolemia A two-sided $\mathrm{P}<0.05$ was considered to indicate a statistically significant difference. Correlations between miRNA expression levels were studied by Spearman analysis. All analyses were performed using SPSS 15.0 software (SPSS, Inc., Chicago, IL, USA).

\section{Results}

Clinical characteristics of patients. Clinicopathological characteristics of the patients enrolled in the study are presented in Table I. The mean age was 65 years and the population consisted of $68 \%$ males. The laboratory data of the patients are presented in Table II.

Relative expression levels of plasma $\mathrm{miR}-208$ a and $\mathrm{miR}-370$ in $C A D$ patients and non-CAD subjects. The relative plasma levels of miR-208a and miR-370 in CAD patients and non-CAD patients were determined. The results are presented in Fig. 1. The median values of miR-208a and miR-370 were 1.57 and 15.21 in the CAD patients, respectively; whereas, in the non-CAD patients, those of miR-208a and miR-370 were 0.12 and 5.67, respectively. Plasma expression levels of miR-208a $(\mathrm{P}=0.006)$ and miR-370 $(\mathrm{P}=0.003)$ were significantly higher in the $\mathrm{CAD}$ group than in the non-CAD group.

Correlations of plasma miR-208a and miR-370 expression levels with blood lipid profiles. The correlation between plasma miR-208a and miR-370 expression levels and blood lipid profiles was analyzed. miR-370 was found to be positively correlated with total cholesterol (TC), triglycerides (TG), low-density lipoprotein cholesterol (LDL-C), but not high-density lipoprotein cholesterol (HDL-C); while miR-208a was not correlated with any of these parameters (Table III).

Diagnostic potential of miR-208a and miR-370 in CAD patients. ROC analysis was performed to examine the potential of miR-208a and miR-370 as biomarkers for CAD patients (Fig. 2). The AUC of miR-208a was 0.819 (95\% CI, 
Table II. Clinical laboratory data of the subjects.

\begin{tabular}{lccc}
\hline Variables & $\begin{array}{c}\text { CAD } \\
\text { patients, } \\
\mathrm{n}=95\end{array}$ & $\begin{array}{c}\text { Non-CAD } \\
\text { subjects, } \\
\mathrm{n}=50\end{array}$ & P-value \\
\hline FBS (mmol/l) & $5.0(4.1-6.9)$ & $5.0(4.1-6.0)$ & 0.365 \\
TG $(\mathrm{mmol} / \mathrm{l})$ & $1.4(0.3-6.1)$ & $1.2(0.3-3.1)$ & 0.411 \\
TC $(\mathrm{mmol} / \mathrm{l})$ & $4.6(1.8-7.5)$ & $4.2(1.6-6.1)$ & 0.214 \\
HDL-C (mmol/l) & $1.1(0.9-1.5)$ & $1.1(1.0-1.5)$ & 0.632 \\
LDL-C (mmol/l) & $2.6(1.5-5.2)$ & $2.4(1.4-4.0)$ & 0.519 \\
AST (U/l) & $23.5(15.3-41.3)$ & $17.6(11.2-34.7)$ & 0.313 \\
ALT (U/l) & $26.0(11.0-54.2)$ & $20.1(11.2-32.7)$ & 0.289 \\
Cr $(\mu \mathrm{mol} / \mathrm{l})$ & $67.2(46.9-98.3)$ & $67.3(47.1-91.4)$ & 0.768 \\
\hline
\end{tabular}

Data are presented as the median with the range in parentheses. CAD, coronary artery disease; FBS, fasting blood sugar; TC, total cholesterol; TG, triglycerides; HDL-C, high-density lipoprotein cholesterol; LDL-C, low-density lipoprotein cholesterol; AST, aspartate aminotransferase; ALT, alanine aminotransferase; $\mathrm{Cr}$, creatinine.

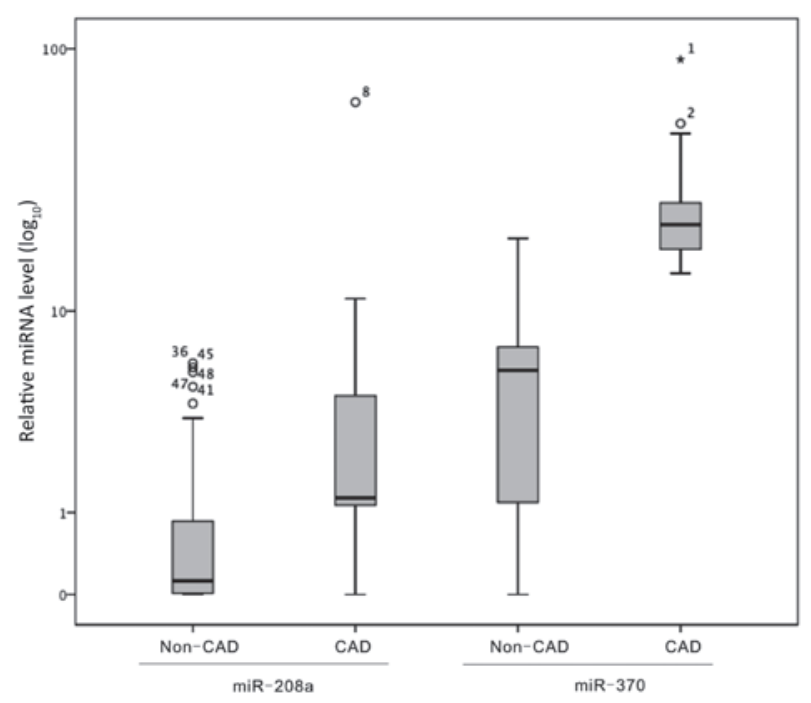

Figure 1. Box plots representing plasma miR-208a and miR-370 expression levels in CAD patients and non-CAD subjects. Expression levels of the miRNAs were normalized to cel-miR-39 and the relative expression values (no unit) are shown on the y-axis. In the boxplot, the upper lines of the boxes indicate the value of the 75th percentile, the lowest lines that of the 25th percentile; and the thick bars indicate the median values of the groups. The circles above the boxes indicate abnormally high or low values in the group. The asterisk indicates an extreme value. The numbers next to the circles and the asterisk are the serial numbers of the subjects with those. CAD, coronary artery disease; mi, micro.

0.739-0.898; $\left.\mathrm{P}=2.97 \times 10^{-7}\right)$. For miR-370, the AUC was 0.745 (95\% CI, 0.667-0.823; $\left.\mathrm{P}=1 \times 10^{-6}\right)$. At the optimal cut-off value of the relative quantification values, the sensitivity of miR-208a was $91.6 \%$ and the specificity was $72.0 \%$; the sensitivity of miR-370 was $55.8 \%$ and the specificity was $92.0 \%$.

Combination of miR-208a and miR-370 exhibited improved $A U C$ values than each individual miRNA. The distribution of plasma levels of miR-208 and miR-370 were different among all of the subjects. Spearman analysis was used to analyze the association between miR-208a and miR-370 expression. There was only a weak, however statistically significant, correlation between miR-208a and miR-370 $\left(r_{s}=0.229 ; \mathrm{P}=0.006\right)$. Combination of these two miRNAs was subjected to ROC analysis. The AUC was 0.856 (95\% CI, 0.796-0.917; $\left.\mathrm{P}=1.89 \times 10^{-9}\right)$. At the optimal cut-off, the sensitivity of the combination of the two miRNAs was $73.7 \%$ and the specificity was $86.0 \%$.

\section{Discussion}

CAD is the most common lethal factor in industrialized countries and poses a marked burden on the public health system (1). The well-determined risk factors of CAD include current smoking habit, hypertension, obesity, diabetes, stress and high blood lipids. miRNAs that are important in these biological processes may have diagnostic potential to discriminate CAD. The miR-208 family (consisting of miR-208a, miR-208b and miR-499) exert critical functions in muscle performance (16). Transgenic overexpression of miR-208a in the heart was identified to be sufficient to induce hypertrophic growth in rodents via inhibiting thyroid hormone-associated protein 1 and myostatin (17). Anti-miR-208a treatment prevented pathological cardiac remodeling and improved cardiac function and survival during hypertension-induced heart failure in Dahl hypertensive rats (18). miRNA distribution analysis revealed that miR-208a was exclusively expressed in the heart, which is important for diagnostic purposes $(19,20)$. The miR-208a expression level (as well as miR-208b and miR-499) was elevated markedly following MI and the miR-208 family has been recommended as a biomarker for MI replacing traditional circulating cardiac troponin I in a number of studies (19,21-23). As for CAD, there are fewer reports regarding miR-208a, and the results are inconsistent. Fichtlscherer et al (11) reported that the expression level of miR-208a tends to be higher in patients with CAD, while Nabiałek et al (24) reported that the miR-208a level was not different between stable CAD patients and healthy control subjects. In the present study, the miR-208a expression level was identified to be significantly higher in CAD patients when compared with non-CAD control subjects. The discrepancy between the current results and those of Nabiałek et al (24) may result from the smaller number of subjects in the previous study (four stable CAD patients and five control subjects).

The main cause of CAD is atherosclerosis, which is a chronic lipid-driven inflammatory disease, with accumulation of lipids on the arterial wall. Hyperlipidemia is a major risk factor for CAD. miR-370 is a key miRNA in lipid metabolism and downregulates the expression of the carnitine palmitoyl transferase $1 \alpha$ gene controlling fatty acid oxidation (25). Motawae et al (26) reported that miR-370 was positively associated with body mass index, LDL-C and LDL-C/HDL-C ratio, and was significantly higher in CAD patients and type 2 diabetes patients with CAD (26). In the present study, miR-370 was found to be positively correlated with TC, TG, LDL-C, and significantly higher in the CAD patients than in the control subjects.

ROC analysis was conducted to investigate the performance of miR-208a and miR-370. miR-208a may be a more efficacious biomarker (AUC $=0.819)$ than miR370 (AUC=0.745). miR-208a exhibits a higher sensitivity (91.6\%) than miR-370 
Table III. Spearman analysis of the correlations of miR-208a and miR-370 with blood lipid profiles.

$\begin{array}{lcccc}\text { Groups } & \text { Total cholesterol } & \text { Triglycerides } & \begin{array}{c}\text { Low-density lipoprotein } \\ \text { cholesterol }\end{array} & \begin{array}{c}\text { High-density lipo } \\ \text { cholestero }\end{array} \\ \begin{array}{l}\text { CAD patients }(\mathrm{n}=95) \\ \text { miR-208a }\end{array} & 0.143 & 0.107 & 0.122 & 0.025 \\ \text { miR-370 } & 0.466^{\mathrm{a}} & 0.421^{\mathrm{b}} & 0.457^{\mathrm{a}} & -0.041 \\ \begin{array}{l}\text { Non-CAD patients (n=95) } \\ \text { miR-208a }\end{array} & 0.137 & 0.126 & & -0.019 \\ \text { miR-370 } & 0.453^{\mathrm{a}} & 0.397^{\mathrm{b}} & 0.461^{\mathrm{a}} & -0.036\end{array}$

Correlations of miR-208a and miR-370 with blood lipid profiles are presented as correlation coefficients (R). ${ }^{\text {a }}<<0.001$ and ${ }^{\mathrm{b}} \mathrm{P}<0.05$, indicating that the $\mathrm{R}$ values are statistically significant. In CAD and non-CAD patients, miR-370 is correlated with blood lipid profiles, while miR-208a is not. CAD, coronary artery disease.
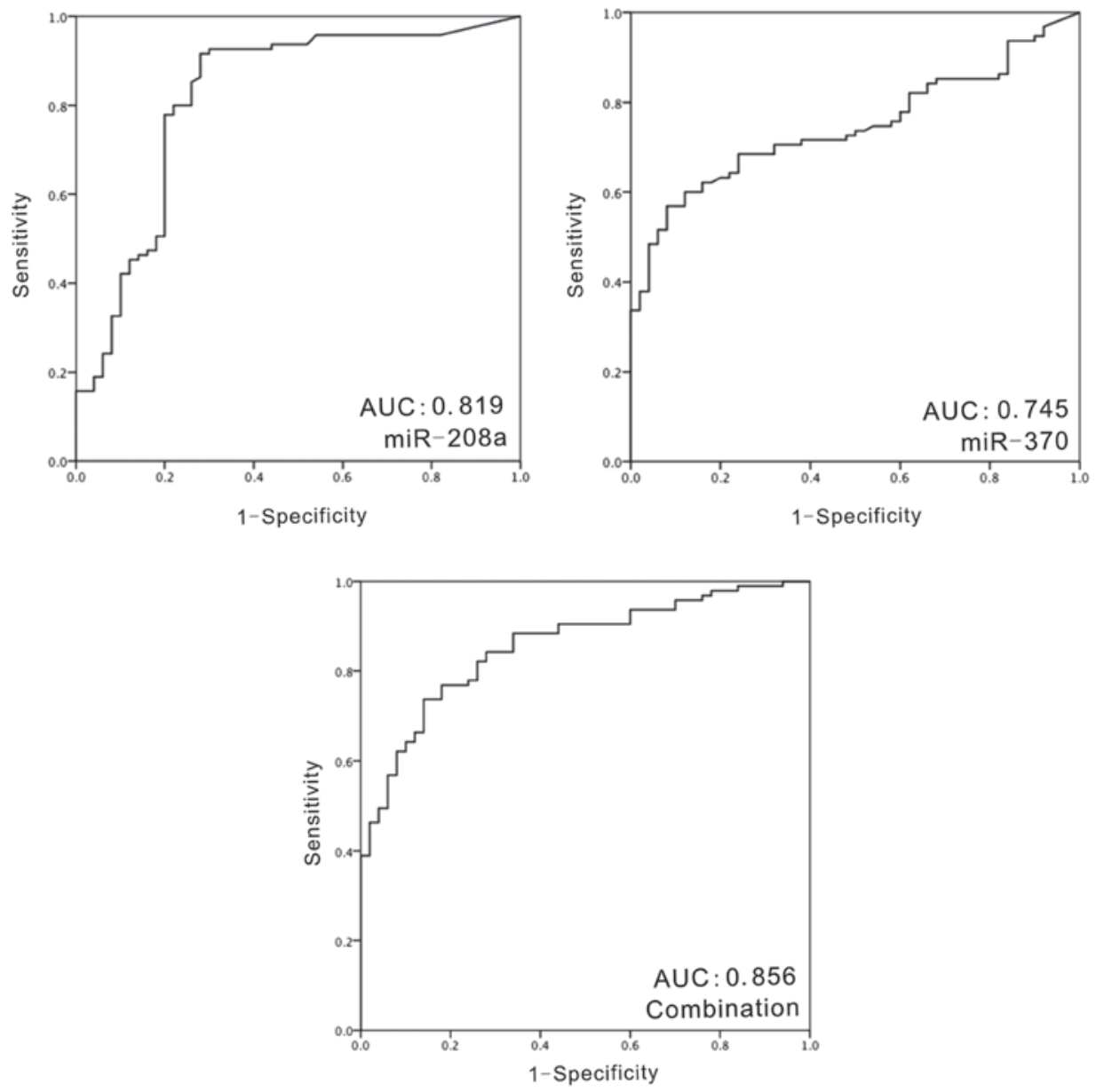

Figure 2. Receiver operating characteristic analysis using miR-208a miR-370 and their combination in CAD patients ( $\mathrm{n}=95)$ and non-CAD controls ( $\mathrm{n}=50$ ). $\mathrm{mi}$, micro; $\mathrm{CAD}$, coronary artery disease; AUC, area under the curve.

(55.8\%); while the specificity of miR-370 was higher $(92.0 \%)$ than that of miR-208a (72.0\%). As the two miRNAs are involved in different biological pathways, it may be better to use the two miRNAs in combination rather than individually. The present study found that the AUC of the combination of miR-208a and miR-380 was largest at 0.856.

In conclusion, expression levels of miR-208a (exclusively expressed in the heart) and miR-370 (vital to lipid metabolism) were significantly higher in CAD patients, rendering them as potential diagnostic tools for discriminating CAD to facilitate with the management of patient care. However, to date no single miRNA could be used as potential biomarker for CAD due to unsatisfactory sensitivity and specificity values. The present study indicates that the combination of miRNAs in different pathways concerning CAD may act more effectively than individual ones for the diagnosis of CAD. However, in the 
current study the sensitivity of the combination (73.7\%) at the optimal cut-off is not satisfactory, as it was lower than that of miR-208a alone. Therefore, further investigations are required to establish suitable miRNAs and their combinations for the early diagnosis of CAD.

\section{Acknowledgements}

The present study was supported by the Medical and Health Science and Technology Development Plan of Shandong Province of China (grant no. 2014WS0516) and Natural Science Foundation of Liaoning Province of China (grant no. 2013023044).

\section{References}

1. Chen WW, Gao RL, Liu LS, Zhu ML, Wang W, Wang YJ, Wu ZS, Li HJ, Zheng Z, Jiang LX, et al: Outline of the report on cardiovascular disease in China, 2014. Chinese Circulation Journal 30: 617-622, 2015 (In Chinese).

2. Goedeke L, Aranda JF and Fernández-Hernando C: MicroRNA regulation of lipoprotein metabolism. Curr Opin Lipidol 25 282-288, 2014.

3. Dangwal S and Thum T: MicroRNA therapeutics in cardiovascular disease models. Annu Rev Pharmacol Toxicol 54: 185-203, 2014.

4. Bartel DP: MicroRNAs: Genomics, biogenesis, mechanism, and function. Cell 116: 281-297, 2004.

5. Creemers EE, Tijsen AJ and Pinto YM: Circulating microRNAs: novel biomarkers and extracellular communicators in cardiovascular disease? Circ Res 110: 483-495, 2012.

6. Gilad S, Meiri E, Yogev Y, Benjamin S, Lebanony D, Yerushalmi N, Benjamin $\mathrm{H}$, Kushnir M, Cholakh $\mathrm{H}$, Melamed N, et al: Serum microRNAs are promising novel biomarkers. PLoS One 3: e3148, 2008.

7. Gidlöf O, Andersson P, van der Pals J, Götberg M and Erlinge D: Cardiospecific microRNA plasma levels correlate with troponin and cardiac function in patients with ST elevation myocardia infarction, are selectively dependent on renal elimination, and can be detected in urine samples. Cardiology 118: 217-226, 2011.

8. Olivieri F, Antonicelli R, Lorenzi M, D'Alessandra Y,Lazzarini R, Santini G, Spazzafumo L, Lisa R, La Sala L, Galeazzi R, et al: Diagnostic potential of circulating miR-499-5p in elderly patients with acute non ST-elevation myocardial infarction. Int J Cardiol 167: 531-536, 2013.

9. Wang GK, Zhu JQ, Zhang JT, Li Q, Li Y, He J, Qin YW and Jing Q: Circulating microRNA: a novel potential biomarker for early diagnosis of acute myocardial infarction in humans. Eur Heart J 31: 659-666, 2010.

10. Ren J, Zhang J, Xu N, Han G, Geng Q, Song J, Li S, Zhao J and Chen H: Signature of circulating microRNAs as potential biomarkers in vulnerable coronary artery disease. PLoS One 8: e80738, 2013.

11. Fichtlscherer S, De Rosa S, Fox H, Schwietz T, Fischer A, Liebetrau C, Weber M, Hamm CW, Röxe T, Müller-Ardogan M, et al: Circulating microRNAs in patients with coronary artery disease. Circ Res 107: 677-684, 2010.
12. Jansen F, Yang X, Proebsting S, Hoelscher M, Przybilla D, Baumann K, Schmitz T, Dolf A, Endl E, Franklin BS, et al: MicroRNA expression in circulating microvesicles predicts cardiovascular events in patients with coronary artery disease. J Am Heart Assoc 3: e001249, 2014.

13. Sondermeijer BM, Bakker A, Halliani A, de Ronde MW, Marquart AA, Tijsen AJ, Mulders TA, Kok MG, Battjes S, Maiwald $\mathrm{S}$, et al: Platelets in patients with premature coronary artery disease exhibit upregulation of miRNA340* and miRNA624*. PLoS One 6: e25946, 2011.

14. Schulte $C$ and Zeller T: MicroRNA-based diagnostics and therapy in cardiovascular disease-summing up the facts. Cardiovasc Diagn Ther 5: 17-36, 2015.

15. Livak KJ and Schmittgen TD: Analysis of relative gene expression data using real-time quantitative PCR and the 2(- $\Delta \Delta \mathrm{C}(\mathrm{T}))$ Method. Methods 25: 402-408, 2001.

16. van Rooij E, Quiat D, Johnson BA, Sutherland LB, Qi X, Richardson JA, Kelm RJ Jr and Olson EN: A family of microRNAs encoded by myosin genes governs myosin expression and muscle performance. Dev Cell 17: 662-673, 2009.

17. Callis TE, Pandya K, Seok HY, Tang RH, Tatsuguchi M, Huang ZP, Chen JF, Deng Z, Gunn B, Shumate J, et al: MicroRNA-208a is a regulator of cardiac hypertrophy and conduction in mice. J Clin Invest 119: 2772-2786, 2009.

18. Montgomery RL, Hullinger TG, Semus HM, Dickinson BA, Seto AG, Lynch JM, Stack C, Latimer PA, Olson EN and van Rooij E: Therapeutic inhibition of miR-208a improves cardiac function and survival during heart failure. Circulation 124: 1537-1547, 2011

19. Ji X, Takahashi R, Hiura Y, Hirokawa G, Fukushima Y and Iwai N: Plasma miR-208 as a biomarker of myocardial injury. Clin Chem 55: 1944-1949, 2009.

20. van Rooij E, Sutherland LB, Qi X, Richardson JA, Hill J and Olson EN: Control of stress-dependent cardiac growth and gene expression by a microRNA. Science 316: 575-579, 2007.

21. Liu X, Fan Z, Zhao T, Cao W, Zhang L, Li H, Xie Q, Tian Y and Wang B: Plasma miR-1, miR-208, miR-499 as potential predictive biomarkers for acute myocardial infarction: an independent study of Han population. Exp Gerontol 72: 230-238, 2015.

22. Huang Y and Li J: MicroRNA208 family in cardiovascular diseases: Therapeutic implication and potential biomarker. J Physiol Biochem 71: 479-486, 2015.

23. Xiao J, Shen B, Li J, Lv D, Zhao Y, Wang F and Xu J: Serum microRNA-499 and microRNA-208a as biomarkers of acute myocardial infarction. Int J Clin Exp Med 7: 136-141, 2014.

24. Nabiałek E, Wańha W, Kula D, Jadczyk T, Krajewska M, Kowalówka A, Dworowy S, Hrycek E, Włudarczyk W, Parma Z, et al: Circulating microRNAs (miR-423-5p, miR-208a and miR-1) in acute myocardial infarction and stable coronary heart disease. Minerva Cardioangiol 61: 627-637, 2013.

25. Iliopoulos D, Drosatos K, Hiyama Y, Goldberg IJ and Zannis VI: MicroRNA-370 controls the expression of microRNA-122 and Cptlalpha and affects lipid metabolism. J Lipid Res 51: 1513-1523, 2010.

26. Motawae TM, Ismail MF, Shabayek MI and Seleem MM: MicroRNAs 9 and 370 association with biochemical markers in T2D and CAD Complication of T2D. PLoS One 10: e0126957, 2015. 